\title{
Permutations of a semigroup that map to inverses
}

\author{
Peter M. Higgins \\ Dept of Mathematical Sciences, University of Essex, CO4 3SQ, UK \\ email: peteh@essex.ac.uk \\ Dedicated to the memory of John M. Howie
}

\begin{abstract}
We investigate the question as to when the members of a finite regular semigroup may be permuted in such a way that each member is mapped to one of its inverses. In general this is not possible. However we reformulate the problem in terms of a related graph and, using an application of Hall's Marriage Lemma, we show in particular that the finite full transformation semigroup does enjoy this property.
\end{abstract}

\section{Introduction and Background}

One of the major subjects of John Howie's research was the full transformation semigroup [11 - 15]. The topic is flavoured very much by whether the base set of the semigroup of mappings is infinite or finite. In this article we continue the latter thread by studying a question that may be asked of any semigroup but which is more natural in the finite regular case and proves particularly interesting for the finite full transformation semigroup.

The author's paper [6] in Semigroup Forum was dedicated to the late John Howie as it represented a continuation of our work in $[8,9,10,11]$ and especially [10] written jointly with John's colleagues Nik Ruskuc and James 
Mitchell from St Andrews University where John worked for most of his career. A feature of the paper was the application of Hall's Marriage Lemma, a theme that also continues here.

Throughout, $S$ denotes a semigroup and $T_{X}, P T_{X}$ the full and partial transformation semigroups respectively on the base set $X$. When $X=X_{n}=$ $\{1,2, \cdots, n\}$ we write $T_{n}$ for $T_{X}$. Following the texts of Howie $[16,17]$ and the author's [7] we denote the set of idempotents of $S$ by $E(S)$ and the set of regular elements of $S$ by $\operatorname{Reg}(S)$; we shall write $(u, v) \in V(S)$ if $u$ and $v$ are mutual inverses in $S$. We shall also denote this as $v \in V(u)$ so that $V(u)$ is the set of inverses of $u \in S$. We extend the notation for inverses to sets $A: V(A)=\bigcup_{a \in A} V(a)$. Standard results on Green's relations, particularly those stemming from Green's Lemma, will be assumed (Chapter 2 of [17], specifically Lemma 2.2.1) and indeed basic facts and definitions concerning semigroups that are taken for granted in what follows are all to be found in Howie's excellent book [17].

Let $C=\left\{A_{i}\right\}_{i \in I}$ be any finite family of finite sets (perhaps with repetition of sets). A set $\tau \subseteq \cup A_{i}$ is a transversal of $C$ (or a system of distinct representatives) if there exists a bijection $\phi: \tau \rightarrow C$ such that $t \in \phi(t)$ for all $t \in \tau$. We shall denote this by $\tau t C$. We also assume Hall's Marriage Lemma in its various forms. In particular $C$ has a transversal if and only if Hall's Condition is satisfied, which says that for all $1 \leq k \leq|I|$, the union of any $k$ sets from $C$ has at least $k$ members (for this and related background see, for example, the text [22]).

Definitions 1.1 Let $S$ be any semigroup and let $F=\left\{f \in P T_{S}: f(a) \in\right.$ $V(a) \forall a \in \operatorname{dom} f\}$. We call $F$ the set of partial inverse matchings of $S$. We call $f \in F$ a permutation matching if $f$ is a permutation of $S$; more particularly $f$ is an involution matching if $f^{2}=\iota$, the identity mapping.

We note that, by definition, $S$ is regular if and only if $S$ has a (full) matching. The word 'matching' here does not necessarily have the connotation of 'mutual pairing' as it does in graph theory but its use in this context is nonetheless convenient. Some other simple observations are:

- An inverse semigroup $S$ has a unique matching $a \mapsto a^{-1}$, which is an involution. 
- A completely regular semigroup $S$ (a semigroup that is a union of its subgroups) is a disjoint union of its maximal subgroups and so $a \mapsto a^{-1}$, where $a^{-1}$ is the (unique) group inverse of $a$, defines an involution matching of $S$.

- The identity mapping is a permutation matching if and only if $S$ satisfies the equation $x=x^{3}$; in particular this is true of bands.

- $S$ has the property that all of its permutations are permutation matchings if and only if $S$ is a rectangular band as that is exactly the class of semigroups where every member is inverse to every other (characterized by either of the equivalent identities $x=x y x$ or $x=x y z)$.

Semigroups with an involution matching $(*)$, which satisfies the additional property that $(a b)^{*}=b^{*} a^{*}$ are called regular ${ }^{*}$ semigroups. They comprise an umbrella class for the classes of inverse semigroups and completely regular semigroups. There are however other collections of regular *-semigroups of interest including that of partition monoids (see for example [3]).

In the remainder of the paper we shall, unless otherwise stated, assume that $S$ is regular and finite. We shall often denote a matching simply by ', so that the image of $a$ is $a^{\prime}$. The matching $(\cdot)^{\prime}$ is then permutative if $S^{\prime}=\left\{a^{\prime}: a \in S\right\}=S$ and $\left(^{\prime}\right)$ is an involution matching if $\left(a^{\prime}\right)^{\prime}=a \forall a \in S$.

We shall work with the family of subsets of $S$ given by $V=\{V(a)\}_{a \in S}$. The members of $V$ may have repeated elements - for example $S$ is a rectangular band if and only if $V(a)=S$ for all $a \in S$. However, we consider the members of $V$ to be marked by the letter $a$, so that $V(a)$ is an unambiguous member of $V$ (strictly, we are using the pairs $\{a, V(a)\},(a \in S)$ ).

Proposition 1.2 For a finite semigroup $S$ the following are equivalent:

(i) $S$ has a permutation matching;

(ii) $S$ is a transversal of $V=\{V(a)\}_{a \in S}$;

(iii) $|A| \leq|V(A)|$ for all $A \subseteq S$.

Proof (i) $\Rightarrow$ (ii) Let $f: S \rightarrow S$ be a permutation matching. Replacing $a$ by $f^{-1}(a)$ in the condition that $f(a) \in V(a)$ gives that $f^{-1}: S \rightarrow S$ is a 
permutation and $a \in V\left(f^{-1}(a)\right)$ for all $a \in S$, which in turn is equivalent to the condition that the function $g: S \rightarrow V$ whereby $g(a)=V\left(f^{-1}(a)\right)$ is a bijection such that $a \in g(a)$ for all $a \in S$ as

$$
a \in g(a) \forall a \in S \Leftrightarrow a \in V\left(f^{-1}(a)\right) \forall a \in S \Leftrightarrow f(a) \in V(a) \forall a \in S .
$$

Hence if $f$ is a permutation matching then $g$ is a required bijection that shows that $S$ is a transversal of $V$.

(ii) $\Rightarrow$ (i) Suppose that $V$ has a transversal $\tau$ so there exists a bijection $g: \tau \rightarrow V$ such that $t \in g(t)$ for all $t \in \tau$. Since $\tau \subseteq S$ and $|S|=|V|$ it follows that $\tau=S$ and so $g: S \rightarrow V$ and $a \in g(a)$ for all $a \in S$. Define $f: S \rightarrow S$ by $f(a)=g^{-1}(V(a))$, which is then a permutation on $S$. Moreover $f(a) \in g(f(a))=V(a)$ and so $f(a)$ is a permutation matching of $S$.

(ii) $\Leftrightarrow$ (iii) As explained above, for any transversal $\tau$ of $V$ we necessarily have $\tau=S$. By Hall's Marriage Lemma a transversal exists if and only if for every subset $A \subseteq \bigcup V(a)=S$, the condition $|A| \leq|V(A)|$ is satisfied.

Example 1.3 A finite regular semigroup with no permutation matching. Consider the 7-element aperiodic completely 0-simple semigroup $S=\{(i, j)$ : $1 \leq i \leq 2,1 \leq j \leq 3\} \cup\{0\}$, where $E(S)=\{(1,2),(1,3),(2,1)\} \cup\{0\}$. Then $V\{(2,2),(2,3)\}=\{(1,1)\}$ and so Hall's Condition is violated on the set $A=\{(2,2),(2,3)\}$, whence, by Proposition $1.2, S$ has no permutation matching. This example is minimal in view of the next result.

Proposition 1.4 Any regular semigroup with fewer than 7 elements has an involution matching.

Proof Suppose that $S$ is a finite regular semigroup with $|S| \leq 6$. If $S$ had just one $\mathcal{D}$-class, then $S$ would be completely simple and so, being a union of groups, would have an involution matching. Suppose then that $S$ is not a union of groups and so has more than one $\mathcal{D}$-class. Then since $S$ is regular but not a union of groups, it follows that $S$ possesses one $\mathcal{D}$ class $D$ with more than one $\mathcal{R}$-class and more than one $\mathcal{L}$-class. It now follows that $|D| \leq 5$ and since $|D|$ is not prime it follows that $D$ is a $2 \times 2$ 'eggbox' with trivial $\mathcal{H}$-classes. Moreover either each $\mathcal{R}$-class and each $\mathcal{L}$ class consists of a pair of elements exactly one of which is idempotent or $D$ has exactly one non-idempotent. In the first case each element in $D$ has a unique inverse. In the 3-idempotent case it is also clear that the 
four members of $D$ may be matched in mutually inverse pairs (with two idempotents self inverse). The remaining $\mathcal{D}$-classes, whether they number 1 or 2, either consist of idempotents or constitute a 2-element group. In any event it follows that an involution matching for $S$ can be found. Therefore if $|S| \leq 6$ then $S$ has an involution matching.

We denote the class of finite regular semigroups with a permutation matching (respectively involution matching) by $\mathcal{M}$ (respectively $\mathcal{N}$ ).

Proposition 1.5 Each of the classes $\mathcal{M}$ and $\mathcal{N}$ is closed under the taking of direct products but not under the taking of regular subsemigroups or homomorphic images.

Proof. Let $S, T \in \mathcal{M}$. Let $S=S^{\prime}=\left\{s^{\prime}: s \in S\right\}$ and $T=T^{\prime}=\left\{t^{\prime}\right.$ : $t \in T\}$ denote permutation matchings of $S$ and of $T$ respectively. For each $(s, t) \in S \times T$ put $(s, t)^{\prime}=\left(s^{\prime}, t^{\prime}\right) \in V((s, t))$ whereupon $\left(^{\prime}\right)$ is a permutation of $S \times T$ that maps each element to one of its inverses. Hence $\mathcal{M}$ is closed under the taking of direct products. We note that if the matchings of $S$ and of $T$ are both involution matchings, then so is the matching ( $\left(^{\prime}\right)$ for $S \times T$, so the same conclusion holds for the class $\mathcal{N}$.

We show in Theorem 2.12 that $T_{n}$ has a permutation matching. By Example 1.3 we know that finite regular semigroups without permutation matchings exist and since any finite semigroup may be embedded in some $T_{n}$, it follows that $\mathcal{M}$ is not closed under the taking of regular subsemigroups. However, consider the following example.

Let $T$ denote the aperiodic completely 0 -simple semigroup $T=\{(i, j)$ : $1 \leq i, j \leq 3\} \cup\{0\}$, where $E(T)=\{(1,2),(1,3),(2,2),(2,3),(3,1)\} \cup\{0\}$. Note that for each $a \in\{(1,1),(2,1)\}$ we have $V(a)=\{(3,2),(3,3)\}$. In particular it follows that $T$ has an involution matching with pairs $(1,1) \mapsto$ $(3,3),(2,1) \mapsto(3,2)$ and with $e \mapsto e$ for each $e \in E(T)$.

We now note that the semigroup $S$ of Example 1.3, which has no permutation matching, is a retract of $T$, meaning that $S$ is a subsemigroup of $T$ and there is a homomorphism of $T$ onto $S$ that fixes each member of $S$. The subsemigroup of $T$ that we identify with $S$ consists of the 7 members $S=\{(2,1),(2,2),(2,3),(3,1),(3,2),(3,3), 0\}$. Note that the relation $\rho=\{((1, j),(2, j)): 1 \leq j \leq 3\}$ is a congruence on $T$ because a pair 
$(1, j) \in E(T)$ if and only if $(2, j) \in E(T)$. A required retraction of $T$ onto $S$ is then defined by $(1, j) \mapsto(2, j)(1 \leq j \leq 3)$, with each member of $S$ fixed. Therefore $T \in \mathcal{N}$ but $S \notin \mathcal{M}$ and $S$ is both a regular subsemigroup of and a homomorphic image of $T$. This completes the proof. 
Theorem 1.6 The following are equivalent for a finite semigroup $S$ :

(i) $S$ has a permutation matching;

(ii) $S$ has a permutation matching that preserves the $\mathcal{H}$-relation (meaning that $\left.\alpha \mathcal{H} \beta \Rightarrow \alpha^{\prime} \mathcal{H} \beta^{\prime}\right)$;

(iii) each principal factor $D_{a} \cup\{0\}(a \in S)$ has a permutation matching;

(iv) each 0-rectangular band $B=D_{a} \cup\{0\} / \mathcal{H}$ has a permutation matching.

Proof (i) $\Leftrightarrow$ (iii) Suppose that $S=S^{\prime}=\left\{a^{\prime}, \cdots\right\}$ is a permutation matching of $S$ and let $D$ denote a $\mathcal{D}$-class of $S$. For each $a \in D$ we have $V(a) \subseteq D$ so that ' $\left.\right|_{D}$ is a permutation of $D$; extending this by $0 \mapsto 0$ gives a permutation matching for the principal factor $D \cup\{0\}$. Conversely, if each principal factor has a permutation matching, the union of these matchings over the set of $\mathcal{D}$-classes yields a permutation matching of $S$.

(i) $\Rightarrow$ (iv) Let $\Lambda$ denote any collection of $\mathcal{H}$-classes within $D=D_{a}$ and let $n$ be the common cardinal of the $\mathcal{H}$-classes within $D$. For each $a \in H \in \Lambda$ we have that $H_{a^{\prime}} \subseteq D\left(a^{\prime} \in V(a)\right)$. Since $S$ has a permutation matching ', it follows that

$$
\begin{aligned}
& \left|\left\{a \in \cup_{H \in \Lambda} H\right\}\right|=n|\Lambda|=\left|\left\{a^{\prime}: a \in \cup_{H \in \Lambda} H\right\}\right| \leq \\
& \left|\bigcup H_{a^{\prime}}\right|\left(a \in \cup_{H \in \Lambda} H\right)=n\left|\left\{H_{a^{\prime}}: a \in \cup_{H \in \Lambda} H\right\}\right|
\end{aligned}
$$

Let us denote the 0-rectangular band $B$ by $(I \times J) \cup\{0\}$ and take any set $A \subseteq B$. The $\mathcal{H}$-classes within $D$ may be indexed by the members $(i, j) \in$ $I \times J$. For each pair of $\mathcal{H}$-classes $H_{i, j}, H_{k, l} \subseteq D$ we have $H_{k, l} \subseteq V\left(H_{i, j}\right)$ or $H_{k, l} \cap V\left(H_{i, j}\right)=\emptyset$. For each $(i, j) \in A$ we have that $(k, l) \in V((i, j))$ if and only if $H_{k, l} \subseteq V\left(H_{i, j}\right)$. Let $\Lambda=\left\{H_{i, j}:(i, j) \in A\right\}$. Cancelling the common term $n$ in (1) yields:

$$
|A \backslash\{0\}|=|\Lambda| \leq\left|\left\{H_{a^{\prime}}: a \in \cup_{h \in \Lambda} H\right\}\right| \leq|V(A \backslash\{0\})| ;
$$

since $V(0)=\{0\}$ in $B$, it follows that $B$ satisfies Hall's Condition and so $B$ has a permutation matching by Proposition 1.2. 
(iv) $\Rightarrow$ (ii) Let $D=D_{a}$ be the $\mathcal{D}$-class of an arbitrary $a \in S$ consisting of $\mathcal{H}$-classes $H_{i, j}$ where the pairs $(i, j)$ form the set $I \times J$ say. Form a bipartite graph $G$, the two defining independent sets of which are two copies, $X$ and $Y$, of $I \times J$ with $(i, j) \in X$ adjacent to $(k, l) \in Y$ exactly when $(k, l) \in V((i, j))$. By Proposition 1.2 applied to the 0 -rectangular band $B$, each subset $A$ of $X$ is collectively adjacent to at least $|A|$ vertices of $Y$. By the Marriage Lemma, it follows that there is a (one-to-one) matching of $X$ into $Y$, which then induces a permutation of the set of $\mathcal{H}$-classes of $D$ to itself, $H \mapsto H^{\prime}$ say in such a way that each $a \in H$ has a unique inverse $a^{\prime} \in H^{\prime}$. The mapping whereby $a \mapsto a^{\prime}$ is then a bijection of $H$ onto $H^{\prime}$.

Now list the $\mathcal{H}$-classes of $D$ as $H_{1}, H_{2}, \cdots$. For each $a_{1} \in H_{1}$ let $\left\{a_{1}^{\prime}\right\}=$ $V(a) \cap H_{1}^{\prime}$ and in this way form a list $\left\{a_{1}^{\prime}, a_{2}^{\prime}, \cdots, a_{n}^{\prime}\right\}$ say of all the members of $H_{1}^{\prime}$. We next select some $H_{2} \neq H_{1}$ and repeat the previous process, thus extending to a list of inverses of the members of $H_{1}^{\prime} \cup H_{2}^{\prime}$ that exhausts this union. Continue until all members of $X$ have been chosen. The final list $D^{\prime}=\left\{a_{1}^{\prime}, a_{2}^{\prime}, \cdots\right\}$ will then be a list of inverses of all the members of $D$, which is to say that $D^{\prime}=D$. Carrying out this procedure with each $\mathcal{D}$-class of $S$ therefore results in a list $S^{\prime}$ of inverses of the members of $S$ that comprises the whole of $S$, that is $S^{\prime}=S$ and $a \mapsto a^{\prime}$ is a permutation matching of $S$ which, by construction, preserves the $\mathcal{H}$-relation.

(ii) $\Rightarrow$ (i) is immediate.

Remarks 1.7 By above, if $S$ has a permutation matching then $S$ has a permutation matching that preserves Green's $\mathcal{H}$ relation (and necessarily preserves the $\mathcal{D}$ relation) but preservation of either the $\mathcal{L}$ or $\mathcal{R}$ relation cannot be guaranteed even for inverse semigroups. To see this take any inverse semigroup that is not a union of groups (for example, the 5 -element combinatorial Brandt semigroup $S=\mathcal{M}\left[1,2,2, I_{2}\right]$, where $I_{2}$ is the $2 \times 2$ identity matrix). Let $a$ be a member of $S$ that does not lie in a subgroup and let $e=a a^{-1}$. We have $a \mathcal{R} e$ but $a^{-1} \mathcal{L} e=e^{-1}$. If $a^{-1} \mathcal{R} e^{-1}$ we would have $a^{-1} \mathcal{H e}$, which is a contradiction as $a^{-1}$ is not a group element. Hence $\mathcal{R}$ (and by symmetry also $\mathcal{L}$ ) is not preserved by the (unique) permutation matching of $S$.

We may ask whether Proposition 1.6 goes through if we replace 'permutation matching' by 'involution matching' throughout. In this case we do see the implications $((\mathrm{i}) \Leftrightarrow$ (iii) $) \Leftarrow(($ ii $) \Leftrightarrow$ (iv) ) but the missing forward 
implication is not clear: to prove that (ii) $\Rightarrow$ (iv) we note that we may regard the non-zero members of $B$ as $\mathcal{H}$-classes of some $\mathcal{D}$-class $D$ of $S$. We now have a well-defined involution of $B$ in the mapping $H_{a} \mapsto H_{a^{\prime}}$ and $0 \mapsto 0$, which maps each member of $B$ to an inverse in $B$. Conversely, given (iv), the involution on $B$ induces an involution by inverses between pairs of $\mathcal{H}$-classes within $D$ so that (iv) implies (ii).

We also do not have an example of a finite regular semigroup $S$ that has a permutation matching $f$ but no involution matching. In any such example, all of the pemutation matchings $f$ must contain an odd cycle that is free of idempotents, the reason being that the set of members of any even cycle may be partitioned into mutually inverse pairs while for any odd cycle that contains an idempotent we may pair an idempotent in the cycle with itself, leaving an even number of members that may be coupled into inverse pairs.

Questions Does $T_{n}$ have a permutation matching? An involution matching?

We shall give an affimative answer to the first question. A theorem of Schein [21] (see [7, Theorem 6.2.4]) is that $T_{n}$ is covered by its inverse subsemigroups. Indeed Schein proved that every $a \in T_{n}$ has a strong inverse $b \in V(a)$, which means that the subsemigroup $\langle a, b\rangle$ is an inverse subsemigroup of $T_{n}$. However, there seems to be no guarantee that these subsemigroups intersect in an inverse subsemigroup, which would allow us to infer that $T_{n}$ does indeed have an involution matching. 


\section{Graph Factor Interpretation}

All graphs $G=(V, E)$ under consideration will be finite graphs, perhaps with loops (but not multiple edges). Recall that a $k$-factor of $G$ is a subgraph $H=\left(V, E^{\prime}\right)$ of $G$ that is regular of degree $k$. The most important cases are the 1-factors and 2-factors. A 1-factor $H$ is also known as a perfect matching and is essentially a set of disjoint edges of $G$ that span $G$, which is to say covers all the vertices of $G$ so that $V(H)=V(G)$. A 2-factor on the other hand is a set of disjoint cycles of $G$ that collectively contain all of the graph's vertices. Since we allow loops in our graphs, we shall here define a 1-factor to be a subgraph of $G$ that is a disjoint set of edges that cover all the vertices. This allows for some of the edges to be loops (which correspond to vertices of degree 2). To allow for 2-cycles in our discussion, we shall be considering 1,2-factors of $G$, which are spanning subgraphs $H$ of $G$ whose components are either edges or cycles.

Definition 2.1 Let $S$ be a semigroup and let $G=G(S)=(S, E)$ be the graph of inverses, which has vertex set $S$ and $u v$ is an (undirected) edge if $(u, v) \in V(S)$.

Remarks 2.2 A graph of inverses $G(S)$ may contain loops: indeed there is a loop at $a \in G(S)$ if and only if $a=a^{3}$. The semigroup $S$ is regular if and only if $G(S)$ has no isolated vertices (vertices of degree 0) and $S$ is an inverse semigroup if and only if each component of $G(S)$ is a single edge (which may be a loop: indeed in general there is a loop at every idempotent). In general it is simple to show that the set of idempotents $E(S)$ of $S$ forms a semilattice if and only if $\operatorname{Reg}(S)$ forms an inverse subsemigroup of $S$. From this it follows that the components of $G(S)$ each consist of at most 2 vertices if and only if $E(S)$ forms a semilattice.

This following type of graph was first introduced by Graham [5] and later independently by Houghton [18] in relation to finite 0-simple semigroups although those authors explicitly worked in terms of the Rees matrix representation of such semigroups. Graham specifically addressed problems concerning maximal nilpotent subsemigroups and maximal idempotent-generated subsemigroups while Houghton's paper is based on the cohomology set of the graph $\Gamma$ over the underlying group $G$ of the Rees matrix semigroup. A modern treatment of Graham's approach appears in the book of Rhodes and Steinberg [20, Section 4.13.2] where the idempotent-generated subsemigroup 
of a Rees matrix semigroup is studied in terms of the topology of this, its incidence graph in order to reveal results about varieties of finite semigroups and general theorems such as that of Fitzgerald that the idempotent-generated subsemigroup of a regular semigroup is itself regular [4].

Definition 2.3 Let $S$ be a finite regular semigroup $S$.

(a) The incidence graph $G H(S)$ is a bipartite graph with independent sets $\mathbf{L}$ and $\mathbf{R}$ of $\mathcal{L}$ - and $\mathcal{R}$-classes respectively of $S$. An edge runs between $L \in \mathbf{L}$ and $R \in \mathbf{R}$ exactly when $L \cap R$ is a group.

(b) A $\mathcal{D}$-component of $G H(S)$ is the subgraph induced by the set of all $\mathcal{L}$ - and $\mathcal{R}$-classes in some $\mathcal{D}$-class $D$ of $S$.

Since $S$ is regular, the set of $\mathcal{L}$-classes within a $\mathcal{D}$-class $D$ of $S$ is collectively adjacent in $G H(S)$ to the set of $\mathcal{R}$-classes within $D$ and vice-versa, so Definition 2.3(b) is symmetric in $\mathcal{L}$ and $\mathcal{R}$. It is not the case that a $\mathcal{D}$ component of $G H(S)$ is necessarily connected but a $\mathcal{D}$-component of $G H(S)$ is a union of components of $G H(S)$.

We shall call a $\mathcal{D}$-class of $S$ square if it comprises an equal number of $\mathcal{L}$ - and $\mathcal{R}$-classes and we shall call the semigroup $S$ itself square if all of its $\mathcal{D}$-classes are square.

Theorem 2.4 A D -component subgraph $C$ of the incidence graph $G H(S)$ of a finite regular semigroup $S$ has a perfect matching if and only if the corresponding $\mathcal{D}$-class $D$ is square and there is an $\mathcal{H}$-class preserving involution matching of the principal factor $D \cup\{0\}$. Overall the graph $G H(S)$ has a perfect matching if and only if $S$ is square and $S$ has an $\mathcal{H}$-class preserving involution matching.

Remarks 2.5 Neither of the latter two conditions of the first statement of the theorem imply the other. For example, the identity mapping is an $\mathcal{H}$-class preserving involution matching for any non-trivial right zero semigroup $S$, the unique $\mathcal{D}$-class of which is then not square. Next, take the the 10-element combinatorial Rees matrix semigroup $S$ the non-zero $\mathcal{D}$-class of which is the $3 \times 3$ array indexed by $\{1,2,3\} \times\{1,2,3\}$ whose idempotents lie in positions $(1, j)$ and $(i, 1)(i, j \in\{1,2,3\})$. Then $S$ is square but $V\{(2,2),(2,3),(3,2),(3,3)\}=\{(1,1)\}$, so that Hall's Condition on the 
family of sets $V(a)$ is violated and therefore, by Proposition 1.2, $S$ has no permutation matching. Note also that in the semigroup $S$ of Example 1.3 there is a matching of the members of $\mathbf{R}$ into those of $\mathbf{L}$ (but not conversely) and again $S$ has no permutation matching.

Proof of Theorem 2.4 We prove the first statement of the theorem from which the second follows by the argument of Theorem 1.6(i) $\Leftrightarrow$ (iii). Suppose there exists a perfect matching for a $\mathcal{D}$-component $C$ of $G H(S)$ corresponding to some $\mathcal{D}$-class $D$ of $S$ and let $\mathbf{L}, \mathbf{R}$, and $\mathbf{H}$ denote the respective collections of $\mathcal{L}$-, $\mathcal{R}$-, and $\mathcal{H}$-classes of $D$. We shall write a typical $\mathcal{H}$-class $H$ as $H=L \cap R$, where $L$ and $R$ are respectively the $\mathcal{L}$ - and the $\mathcal{R}$-class of $D$ that contain $H$. Let $f: \mathbf{L} \rightarrow \mathbf{R}$ denote a perfect matching of the $\mathcal{D}$-component $C$ of $G H(S)$ corresponding to $D$. It follows at once that $|\mathbf{L}|=|\mathbf{R}|$ and so $D$ is square.

Now introduce the mapping ' $: \mathbf{H} \rightarrow \mathbf{H}$ by $H^{\prime}=(L \cap R)^{\prime}=R f^{-1} \cap L f$. By definition of the graph $G H(S)$, we have that $L \cap L f$ and $R f^{-1} \cap R$ are group $\mathcal{H}$-classes and so $H^{\prime}$ is a set of inverses of $H$. (Indeed there is a perfect matching between $H$ and $H^{\prime}$ in the graph of inverses $G(S)$, although $H=H^{\prime}$ is possible in which case some of the edges of this matching may be loops.) Note that the mapping ' is an involution (and in particular a bijection) on $\mathbf{H}$ for we have:

$$
\left(H^{\prime}\right)^{\prime}=\left((L \cap R)^{\prime}\right)^{\prime}=\left(R f^{-1} \cap L f\right)^{\prime}=(L f) f^{-1} \cap\left(R f^{-1}\right) f=L \cap R=H .
$$

Therefore we may define an $\mathcal{H}$-class preserving involution matching, which we shall also denote by ', on the principal factor of $D$ by taking an arbitrary $\mathcal{H}$-class $H \in \mathbf{H}$ and an arbitrary member $h \in H$ and defining $h \mapsto h^{\prime}$ where $h^{\prime} \in H^{\prime}$ is the unique inverse of $h$ that is to be found in $H^{\prime}$. This completes the proof of the forward implication of the first statement of the theorem.

Conversely suppose that the principal factor of the $\mathcal{D}$-class $D$ of $S$ is square so that $|\mathbf{L}|=|\mathbf{R}|=n$ say, where $\mathbf{L}$ and $\mathbf{R}$ once again denote the respective collections of $\mathcal{L}$ - and of $\mathcal{R}$-classes contained in $D$, which then forms an $n \times n$ array of the $\mathcal{H}$-classes that comprise the collection $\mathbf{H}$. Let us suppose further that the principal factor of $D$ has an $\mathcal{H}$-class preserving involution matching ' $: D \cup\{0\} \rightarrow D \cup\{0\}$. Let $T$ be any collection of $k$ say members of $\mathbf{L}$. The the union of the members of $T$ is the union of $n k$ $\mathcal{H}$-classes of $D$, which is mapped by the bijection ' onto a set of $n k \mathcal{H}$-classes of $D$. Since each member of $\mathbf{R}$ contains exactly $n \mathcal{H}$-classes, it follows that 
$T^{\prime}$ meets at least $k$ of the $\mathcal{R}$-classes of $D$. Now for each such $\mathcal{R}$-class $R_{1}$ that meets $T^{\prime}$ there exists some $\mathcal{H}$-class $H_{1}$ contained in some $\mathcal{L}$-class $L_{1} \subseteq T$ such that $H_{1}^{\prime} \subseteq R_{1}$. From this it follows that $L_{1} \cap R_{1}$ is a group so that $L_{1} R_{1}$ is an edge in $G H(S)$ whence each such $R_{1}$ is adjacent to some member of $T$. Hence the set of $k \mathcal{L}$-classes that form $T$ is collectively adjacent to at least $k \mathcal{R}$ classes of $D$. It follows by Hall's Lemma that there is a matching of $\mathbf{L}$ into $\mathbf{R}$ in the $\mathcal{D}$-component $C$ of $G H(S)$ corresponding to $D$, which, since $|\mathbf{L}|=|\mathbf{R}|$, is a perfect matching of the independent sets $\mathbf{L}$ and $\mathbf{R}$ within each $\mathcal{D}$-class $D$.

Examples 2.6 An example that illustrates Theorem 2.4 is provided by the semigroup of all orientation-preserving mappings $O P_{n}$ on an $n$-cycle $c=(01 \cdots n-1)$, which are the members $\alpha \in T_{n}$ such that the sequence $(0 \alpha, 1 \alpha, \cdots,(n-1) \alpha)$ is cyclic. As shown in [1], $O P_{n}$ is a finite regular monoid and the kernel classes of $\alpha \in O P_{n}$ are convex (that is form intervals of the underlying cycle). Hence the set of kernel classes (and hence the $\mathcal{R}$-class) of a mapping $\alpha \in O P_{n}$ of rank $k \geq 2$ is determined by a strictly ascending list $P$ of $k$ integers $P=\left(p_{0}<p_{1}<\cdots<p_{k-1}\right)$; the $p_{i}$ are drawn from the integer interval $[n]=\{0,1, \cdots, n-1\}$ and each $p_{i}$ equals the initial member of a kernel class of $\alpha$ when read in cylic order. Since each $\mathcal{L}$-class of $O P_{n}$ can also be identified with the common image of its members, which corresponds to a $k$-set $A$ drawn from [n], it follows that there is a perfect matching for each $\mathcal{D}$-component of $G H\left(O P_{n}\right)$ corresponding to a $\mathcal{D}$-class of rank $k \geq 2$, defined by matching the $\mathcal{L}$-class $L \in \mathbf{L}$ with the $\mathcal{R}$-class $R \in \mathbf{R}$ that arises by taking the common range $A$ of members of $L$ and letting $A$ act as the set $P$ of initial members of kernel classes of the kernel partition that defines $R$.

By our theorem, it follows that the $\mathcal{D}$-class $D$ of $O P_{n}$ of rank $k \geq 2$ is square (for $D$ we have $|\mathbf{L}|=|\mathbf{R}|=\left(\begin{array}{l}n \\ k\end{array}\right)$ ) and the corresponding principal factor $D \cup\{0\}$ has an $\mathcal{H}$-class preserving involution matching '. We can identify this involution explicitly as follows. Let $\alpha \in O P_{n}$ be a mapping of rank $k \geq 2$ and let $\alpha$ lie in the $\mathcal{H}$-class $L \cap R$ say with $A=\left\{a_{0}<a_{1}<\cdots<a_{k-1}\right\}$ representing the common image set of members of $L$ and let $P=\left\{p_{0}<p_{1}<\right.$ $\left.\cdots<p_{k-1}\right\}$ represent the common set of initial members of kernel classes of $R$. Furthermore, list the kernel classes of $R$ as $K_{0}, K_{1}, \cdots, K_{k-1}$, where $p_{i}$ is the initial member of $K_{i}(0 \leq i \leq k-1)$. The mapping $\alpha \in H$ is then specified by the choice of an integer $r \in[k]$ where $K_{i} \alpha=a_{i \cdot c^{r}}$, where $c=c_{k}$ is the cycle $(01 \cdots k-1)$. The canonical inverse $\alpha^{\prime}$ of $\alpha$ given by 
the involution matching of our theorem is then the mapping $\beta$ with $\mathcal{L}$-class determined by the set $P$ and $\mathcal{R}$-class determined by $A$ (so that the roles of the sets $A$ and $P$ are interchanged when passing form $\alpha$ to $\beta$ ) and with $r$ replaced by $k-r$. Hence if we identify $\alpha \in O P_{n}$ with the triple $\alpha=(A, P, r)$ then $\beta=(P, A, k-r)$. It is now clear that the mapping $\alpha \mapsto \beta$ is an $\mathcal{H}$-class preserving involution on $D$ (as an $\mathcal{H}$-class is determined by the pair $(A, P)$ ) and we may check that $\beta \in V(\alpha)$ as follows. Let $t \in[n]$ with $t \in K_{i}$ say so that the initial member of $K_{i}$ is $p_{i}$. Then we obtain:

$$
t \alpha \beta \alpha=p_{i} \alpha \beta \alpha=a_{i \cdot c^{r}} \beta \alpha=p_{\left(i \cdot c^{r}\right) \cdot c^{k-r}} \alpha=p_{i \cdot c^{k}} \alpha=p_{i} \alpha=t \alpha,
$$

so that $\alpha \beta \alpha=\alpha$ and in the same way we have $\beta=\beta \alpha \beta$, whence $\beta \in$ $V(\alpha)$ and we may denote $\beta$ by $\alpha^{\prime}$. We conclude that $\alpha \mapsto \alpha^{\prime}$ is an $\mathcal{H}$-class preserving involution matching on $D$.

The $\mathcal{D}$-class of all mappings of rank 1 in $O P_{n}$ consists of idempotents (indeed it comprises the set of all right zeros of $O P_{n}$ ), which has an $\mathcal{H}$ class preserving involution matching in the identity mapping. By taking the union of these matching across all $\mathcal{D}$-classes of $O P_{n}$, we obtain an $\mathcal{H}$-class preserving involution matching of $O P_{n}$.

An example of a different kind is provided by the full linear monoid $M_{n}(F)$ of all $n \times n$ matrices over a finite field $F$. This monoid also satisfies the conditions of Theorem 2.4, and thus also admits an involution matching.

Proposition 2.7 The semigroup $S$ has an involution matching (respectively a permutation matching) if and only if $G=G(S)$ has a 1-factor (respectively a 1,2-factor).

Proof. Let ' $: S \rightarrow S$ be an involution matching of $S$. Then the set $M=\left\{a a^{\prime}: a \in S\right\} \subseteq E(G)$. We then see that the subgraph $H$ of $G$ defined by the set of edges $M$ is a 1-factor of $G$ as follows. Since' has domain $S$, then $V(H)=S$. Any walk of length 2 in $H$ has the form $a \rightarrow a^{\prime} \rightarrow\left(a^{\prime}\right)^{\prime}$ but since $\left(a^{\prime}\right)^{\prime}=a$, this walk involves only a single edge, whence $M$ comprises a set of disjoint edges. Conversely, let $H$ be a 1-factor of $G(S)$. Then each vertex $a \in G$ lies on a unique edge $a a^{\prime}$ of $G$ (with $a^{\prime}=a$ possible) whence, by definition of $G(S), a \mapsto a^{\prime}$ is an involution matching of of $S$.

Next suppose that $S$ has a permutation matching ' $: S \rightarrow S$, which can then be regarded as a set of disjoint (oriented) cycles, edges, and fixed 
points, the vertices of which cover $G(S)$. The underlying set of loops (for 1cycles), edges (for 2-cycles) and cycles then represents a 1,2-factor of $G(S)$. Conversely, suppose that $H$ is a 1,2-factor of $G(S)$. Choose any orientation for each of the cycles of $H$ and treat each edge component of $H$ as a 2-cycle, thereby defining a permutation $a \mapsto a^{\prime}$ of $S$ such that $a^{\prime} \in V(a)$, which is by definition a permutation matching of $S$.

The main aim of this section is to show that the finite full transformation semigoup $S=T_{n}$ on the base set $X_{n}=\{1,2, \cdots, n\}$ has a permutation matching, which by Proposition 2.7 is the same as saying that the graph of inverses, $G(S)$ has a 1,2-factor. We reformulate the notion of 1,2-factor in terms of a certain bipartite graph $G^{\prime}$ known as the bipartite double cover of $G$, also known as the canoncial cover or sometimes the Kronecker cover as $G^{\prime}$ is realised as a certain product of $G$ with the single edge $K_{2}$. The original source of this construction seems to be the paper [2].

Proposition 2.8 Let $G=(V, E)$ be a graph (with loops) and let $V^{\prime}$ be a disjoint copy of the vertex set $V$. Let $G^{\prime}$ be the bipartite graph with independent sets $V$ and $V^{\prime}$ with $u v^{\prime} \in E\left(G^{\prime}\right)$ if and only if $u v \in E(G)$. Then $G$ has a 1,2 -factor if and only if $G^{\prime}$ has a 1 -factor.

Proof Suppose that $G^{\prime}$ has a 1-factor $H$. For each $u \in V(G)$ let $v^{\prime} \in$ $V\left(G^{\prime}\right)$ be such that $u v^{\prime} \in E(H)$. Beginning with an arbitrary vertex $u=$ $u_{0} \in V(G)$ we may form a path $u_{0} \rightarrow u_{1} \rightarrow u_{2} \rightarrow \cdots$ in $G$ by defining for $i \geq 1, u_{i}=v$ where $u_{i-1} v^{\prime}$ is the unique edge from $u_{i-1}$ in $H$. There is then a least value of $i \geq 0$ such that for some $j \geq 1$ we have $u_{i}=u_{i+j}=v$, say. If $i \geq 1$ we would have that $u_{i-1} v^{\prime}$ and $u_{i+j-1} v^{\prime}$ were distinct edges of $H$ with a common vertex, contrary to $H$ being a 1-factor of $G^{\prime}$. Hence $u_{i+j}=u_{0}$, giving either a cycle $C$ in $G$ containing $u_{0}$ or a walk of the form $u_{0} u_{1} u_{0}$. Suppose recursively that we have constructed a set of cycles and edges of $G$ : $C_{1}, C_{2}, \cdots$ that are pairwise disjoint. Suppose there remains a vertex $x=x_{0}$ of $V(G)$ that is not a vertex of any of the $C_{i}$. Form a new cycle or edge in $G$ : $x_{0} \rightarrow x_{1} \rightarrow \cdots \rightarrow x_{t}(t \geq 0)$ as above. Suppose that some vertex $x_{i}(1 \leq i)$ is such that $x_{i}=u_{j}$, where $u_{j} \in V(C)$ where $C$ is one of the cycles or edges already in our list of cycles of $G$. Take $i$ to be the least index for which this is true. Then in $G^{\prime}$ we have $x_{i-1} x_{i}^{\prime}$ and $u_{j-1} u_{j}^{\prime}$ are both edges of $H$ but $x_{i}^{\prime}=u_{j}^{\prime}$ while $x_{i-1} \neq u_{j-1}$, contradicting that $H$ is a 1 -factor of $G^{\prime}$. Hence this eventuality does not arise and the process halts with a required list of pairwise disjoint cycles and edges of $G$ that collectively span $G$. 
Conversely, suppose that $G$ has a 1,2-factor $H$, which then comprises a set of pairwise disjoint cycles and edges $C_{1}, C_{2}, \cdots$ of $G$ that cover all of $V(G)$. Choose an orientation for each cycle $C_{i}$, which then induces a mapping ' $: V(G) \rightarrow V(G)$ whereby $u \mapsto v^{\prime}$, where $v$ is the unique vertex that follows $u$ in the oriented cycle that contains the vertex $u$, or $v$ is the other vertex of the edge if $C_{i}$ is just an edge. The corresponding set of edges $u v^{\prime}$ of $G^{\prime}$ then comprise a 1 -factor of $G^{\prime}$.

Lemma 2.9 Let $G$ be a bipartite graph defined on two non-empty disjoint independent sets $X$ and $Y$. Suppose that $X=\bigcup X_{i}$ and $Y=\bigcup Y_{i}$, where both unions are disjoint and comprise an equal number of $t \geq 1$ sets. Define the bipartite subgraphs $G_{i}$ of $G$ by saying that $G_{i}$ has the pair of independent sets $\left(X_{i}, Y_{i}\right)$ where $x \in X_{i}$ is adjacent to $y \in Y_{i}$ if $x y$ is an edge of $G$. Then $G$ has a perfect matching if each of the graphs $G_{i}$ has an $m=m_{i}$-factor for some integer $m_{i}$.

Proof It suffices to check that each $G_{i}$ has a perfect matching for, given this, the union of these perfect matchings will be the required perfect matching for $G$. For a given $G_{i}$, let $H$ be a regular subgraph of $G_{i}$ that spans $G_{i}$ so that each vertex of $H$ has degree $m \geq 1$ say. Take any subset of $k$ vertices of $X_{i}$, which are collectively adjacent to $l$ say vertices in $Y_{i}$. Hence there are at least $k m$ edges in $H$ incident with these $l$ vertices. Since every vertex in $H$ has degree $m$, it follows that $l m \geq k m \Rightarrow l \geq k$. It therefore follows by Hall's Marriage Lemma that $G_{i}$ has a perfect matching. (It follows also that each $\left|X_{i}\right|=\left|Y_{i}\right|$ and $|X|=|Y|$.)

Recall the basic structure of Green's relations for $T_{n}$, which are that $\alpha \mathcal{L} \beta$ if and only if $X \alpha=X \beta, \alpha \mathcal{R} \beta$ if and only if $\operatorname{ker} \alpha=\operatorname{ker} \beta, \alpha \mathcal{D} \beta$ if and only if $|X \alpha|=|X \beta|$ with $\mathcal{D}=\mathcal{J}$. We shall write Ker $\alpha$ for the partition of $X$ induced by ker $\alpha$. Since $\mathcal{H}=\mathcal{R} \cap \mathcal{L}$ it follows that an $\mathcal{H}$-class $H$ of $S=T_{n}$ is characterised by a kernel-range pair $(\Pi, Y)$ where $Y \subseteq X$ and $\Pi$ is a partition of $X$ with $|Y|=|\Pi|=k$ say, $(1 \leq k \leq n) ; k$ is the common rank of members in the $\mathcal{D}$-class containing $H$.

It is convenient here however to introduce a new equivalence relation $\mathcal{Q}$ on $T_{n}$ that lies between $\mathcal{R}$ and $\mathcal{D}$.

Definition 2.10 For $\alpha \in T_{n}$ let us write Ker $\alpha=\left\{K_{1}, K_{2}, \cdots, K_{k}\right\}$ where the kernel classes of $\alpha$ are listed in ascending order of cardinality. Let 
$P_{\alpha}=\left(p_{i}\right)_{1 \leq i \leq k}$, where $p_{i}=\left|K_{i}\right|$. We shall say that $\alpha \mathcal{Q} \beta$ in $T_{n}$ if $P_{\alpha}=P_{\beta}$. We shall write $Q_{\alpha}$ for the $\mathcal{Q}$-class of $\alpha$.

Consider the double bipartite cover $G^{\prime}$ of the graph of inverses $G$ of $S=T_{n}$. For each $\mathcal{Q}$-class $Q_{\alpha}$ of $T_{n}$, let $G_{\alpha}$ be the bipartite subgraph on the independent sets $Q_{\alpha}, Q_{\alpha}^{\prime}$ where $u v^{\prime} \in E\left(G_{i}\right)$ if $u, v \in Q_{\alpha}$ and $u v \in G$. Our main result will follow once the next fact is proved.

Lemma 2.11 Each of the graphs $G_{\alpha}$ as given above is $m$-regular for some positive integer $m=m_{\alpha}$ that depends on $G_{\alpha}$. In particular, $G_{\alpha}$ is itself an $m$-factor of itself.

Theorem 2.12 The semigroup $S=T_{n}$ has a permutation matching.

Proof The $\mathcal{Q}$-classes of $S$ and their dashed counterparts partition the independents sets $S$ and $S^{\prime}$ that together comprise the vertex set of $G^{\prime}$ into an equal number of sets. The bipartite graphs $G_{\alpha}$ based on the independent sets $Q_{\alpha}$ and $Q_{\alpha}^{\prime}$ defined above are then in accord with the hypotheses of Lemma 2.9. Assuming the truth of Lemma 2.11, we may invoke Lemma 2.9 to conclude that $G^{\prime}$ has a 1-factor, whence by Proposition 2.8, $G(S)$ has a 1,2-factor. But then by Proposition 2.7, we conclude that $T_{n}$ has a permutation matching.

Lemma 2.13 Let $H_{1}, H_{2}$ be any two $\mathcal{H}$-classes of $T_{n}$ defined by the respective kernel-range pairs $\left(\Pi_{1}, Y_{1}\right)$ and $\left(\Pi_{2}, Y_{2}\right)$. Then each $\alpha \in H_{1}$ has a (unique) inverse $\beta \in H_{2}$ (and vice versa) if and only if $Y_{1} t \Pi_{2}$ and $Y_{2} t \Pi_{1}$.

Proof Quite generally by standard semigroup theory (consequences of Green's Lemma), each member of $H_{1}$ will be an inverse of some unique member of $\mathrm{H}_{2}$ and vice versa if and only if the $\mathcal{H}$-classes defined by the kernel-range pairs $\left(\Pi_{2}, Y_{1}\right)$ and $\left(\Pi_{1}, Y_{2}\right)$ are groups, which in turn occurs if and only if the given transversal conditions are satisfied.

Proof of Lemma 2.11. Let $\alpha \in T_{n}$. We need to show that the cardinal of $I=\left\{\beta \in T_{n}: \beta \in V(\alpha) \cap Q_{\alpha}\right\}$ is a positive integer $m$ that is independent of the choice of representative of $Q_{\alpha}$. By Lemma 2.13, we may construct all members of $I$ as follows. Since we require that $\beta \in Q_{\alpha}$ we begin with a list of 'boxes' (named sets, the members of which are yet to be specified) $L_{1}, L_{2}, \cdots, L_{k}$ where $k=|X \alpha|$, which are to become the list of kernel classes 
of our mapping $\beta$. All members of $Q_{\alpha}$ such that $X \alpha$ is a transversal of the sets $L_{i}$ are formed by assigning exactly one member of $X \alpha$ to each of the sets $L_{i}$ and then assigning all members of $X \backslash X \alpha$ to the sets $L_{i}$ so that $\left|L_{i}\right|=p_{i}$. Let us denote the number of ways in which this can be done by $l$, noting that $l \geq 1$. We are not asserting that the two stages of assigning the members of $X \alpha$ to the $L_{i}$ and then assigning the remaining members of $X$ to the $L_{i}$ are combinatorially independent: indeed completion of the two stages will sometimes fail to yield unique outcomes exactly when there is repetition among the integers $p_{i}$. However, the value of $l$ depends only on $Q_{\alpha}$ and not on the choice of $\alpha$ as $l$ depends only on the cardinals of the sets $L_{i}$ and the integer $k$ and not on the membership of $X \alpha$. The number $l$ is then the number of partitions $\Pi$ of $X_{n}$ that correspond to kernel partitions of the members of $Q_{\alpha}$ for which $X \alpha$ is a transversal of $\Pi$.

All members of $I$ can now be formed as follows. Take any of the $l$ partitions above to act as $\operatorname{Ker} \beta$. To ensure that $\beta \in V(\alpha)$ we need to take $X \beta$ to be any transversal of Ker $\alpha$. Again the number of choices $r$ available depends only on $P_{\alpha}$ and is independent of the the choice of representative of $Q_{\alpha}$; indeed it is easy to see that $r=p_{1} p_{2} \cdots p_{k} \geq 1$. The required number $m=l r \geq 1$ is then, by Lemma 2.13 , the cardinal of the set $I$. Therefore the subgraph $G_{i}$ in question is regular of degree $m \geq 1$, as required to complete the proof Lemma 2.11, and hence also of Theorem 2.12.

Remarks 2.14 We note our proof shows additionally that $T_{n}$ has a permutation matching $\phi$ the restriction of which to each $\mathcal{Q}$-class of $T_{n}$ is also a permutation. We may also insist that $\phi$ simultaneously preserves the $\mathcal{H}$-relation. To see this, observe that for each $\mathcal{Q}$-class $Q$ in $T_{n}$, the set $Q \cup\{0\}$ forms a subsemigroup (indeed a right ideal) of the corresponding principal factor $D \cup\{0\}$. We may therefore work through the proof of Theorem 1.6 with the semigroups $Q \cup\{0\}$ in place of the principal factors and $\mathcal{Q}$-preserving permutation matchings throughout and so construct a permutation matching for $T_{n}$ that preserves both $\mathcal{Q}$ - and $\mathcal{H}$-classes.

We note that the semigroup generated by a fixed $\mathcal{Q}$-class has been studied previously by Levi and her co-authors in several papers: see for example [19].

A similar proof yields that the partial transformation semigroup $P T_{n}$, which is isomorphic to the subsemigroup of all mappings that fix 0 in $T_{X}$ where $X=\{0,1,2, \cdots, n\}$, also has a permutation matching. We just need 
to run through the previous arguments, restricting ourselves throughout to mappings that fix 0 .

ACKNOWLEDGEMENT: The author would like to thank an anonymous referee of this paper for some enlightening observations that led to its improvement.

\section{References}

[1] Catarino, P.M. and P.M. Higgins, The monoid of Orientation-preserving Mappings on a chain, Semigroup Forum, Vol. 58 (1999) 190-206.

[2] Dulmage, A.L. and N.S. Mendelsohn, Coverings of bipartite graphs, Canadian Journal of Mathematics 10: 517-534 (1958).

[3] East, J. and D. FitzGerald, The semigroup generated by the idempotents of a partition monoid, Journal of Algebra, Volume 372, 108-133 (2012).

[4] Fitzgerald, D., On inverses of products of idempotents in regular semigroups, J. Australian Mathematical Society, Series A, Vol. 13, 335-337 (1972).

[5] Graham, R.L., On Finite 0-simple Semigroups and Graph Theory, Mathematical Systems Theory, Vol. 2, No. 4 (1968), 325-339.

[6] Higgins, P.M., The product of the idempotents and an $\mathcal{H}$-class of the finite full transformation semigroup, Semigroup Forum (2012) 84: 203215 .

[7] Higgins, P.M., Techniques of semigroup theory, OUP (1992).

[8] Higgins, P.M., J.M. Howie, and N. Ruskuc, Generators and factorisations of transformation semigroups, Proc. Roy. Soc. of Edinb., 128A, 1355-1369, (1998).

[9] Higgins, P.M., J.M. Howie, J.D. Mitchell and N. Ruskuc, Countable versus uncountable ranks in infinite semigroups of transformations and relations, Proc. Edin. Math. Soc. (2003), 46, 531-544.

[10] Higgins, P.M., J.M. Howie and N. Ruskuc, Set products in transformation semigroups, Proc. Roy. Soc. Edin, 133A, 1121-1136 (2003). 
[11] Howie, J.M., N. Ruskuc and P.M. Higgins, On the relative ranks of full transformations semigroups, Comm. in Alg, 26(3), 733-748 (1998).

[12] Howie, J.M., The subsemigroup generated by the idempotents of a full transformation semigroup, J. Lon. Math. Soc., 41 (1966), 707-716.

[13] Howie, J.M., Products of idempotents in certain transformation semigroups, Proc. Roy. Soc. Edinb. 17, 223-236 (1971).

[14] Howie, J.M., Idempotent generators in finite full transformation semigroups, Proc. Roy. Soc. Edinb. A 81, 317-23 (1978).

[15] Howie, J.M., Some subsemigroups of infinite full transformation semigroups, Proc. Roy. Soc. Edin, 88A, 159-167, (1981).

[16] Howie, J.M., An introduction to semigroup theory, Academic Press, London, (1976).

[17] Howie, J.M., Fundamentals of semigroup theory, Clarendon Press (1995).

[18] Houghton, C.H. Completely 0-simple semigroups and their associated graphs and groups, Semigroup Forum, Vol. 14 (1977) 41-67.

[19] Levi I. and S. Seif, Constructing techniques for labeling constant weight Gray codes with applications to minimal generating sets of semigroups, J. Algebra, 266(1): 189-219, (2003).

[20] Rhodes, J. and B. Steinberg, The q-theory of semigroups, SpringerVerlag, New York (2009).

[21] Schein, B.M., A symmetric semigroup of transformations is covered by its inverse subsemigroups, Acta. Math. Acad. Sci. Hung.22, 163-71 (1971).

[22] Wilson, R.J., Introduction to graph theory, 5th edition, (Pearson Education Ltd, United States, 2010). 